\title{
Analysis of Knowledge Management in Industrial Sectors by Means of Neural Models
}

\author{
Álvaro Herrero, Emilio Corchado, Lourdes Sáiz-Bárcena \\ and Miguel A. Manzanedo
}

\begin{abstract}
It is required for an organization, before successfully applying a Knowledge Management (KM) methodology, to develop and implement a knowledge infrastructure, consisting of people, organizational and technological systems. Up to now, few approaches have been proposed for such technological systems supporting KM in organizations. Present paper advances previous work by proposing neural projection models for the analysis of the KM status of companies from two different industrial sectors. Exploratory methods are applied to real-life case studies to know and understand the structure of KM data. Subsequently, the application of such models generates meaningful conclusions that allow experts to diagnose KM from two different points of view: companies on the one hand and industrial sectors on the other hand.
\end{abstract}

Keywords Knowledge management • Unsupervised neural networks • Exploratory projection pursuit

\footnotetext{
Á. Herrero (®) · L. Sáiz-Bárcena · M.A. Manzanedo

Department of Civil Engineering, University of Burgos, Avenida de Cantabria s/n, 09006 Burgos, Spain

e-mail: ahcosio@ubu.es

L. Sáiz-Bárcena

e-mail: 1saiz@ubu.es

M.A. Manzanedo

e-mail: mmanz@ubu.es

E. Corchado

Departamento de Informática y Automática, Universidad de Salamanca,

Plaza de la Merced s/n, 37008 Salamanca, Spain

e-mail: escorchado@usal.es
} 


\section{Introduction}

Knowledge Management (KM) [1] means that organizations can capture and share the collective experience and the know-how (knowledge) of their employees and apply their knowledge in intelligent ways [2]. In an environment such as today's, where everything changes at great speed and almost nothing remains static, it could be said that knowledge emerges as the key factor in any economy [3]. A firm requires both "general" knowledge and "specific/singular" knowledge, which will permit the firm to pursue excellence alongside others. This class of first-level knowledge is held by a small number of people, without forgetting that more select and sophisticated knowledge is required, if possible, in pioneering firms, such as those analyzed in this study, in an area where the offer is, unfortunately, further and further away from satisfying demand.

Companies from different industrial sectors in Burgos (Spain) are analyzed in present study. These companies are in a dynamic environment characterized by: high levels of competitiveness; clients with increasing demands that know their needs and how to satisfy them; a need for personalized products and services; the existence of novel techniques that require professionals to have a knowledge and a good command; highly-qualified providers; disconcerting changes and new problems that must be addressed.

Under such circumstances, traditional sources for competitive advantages (such as physical, financial and technological assets, access to raw material or special markets, and list of clients) are not enough because these sources are available for the majority of companies, subject to the same conditions.

To effectively compete, companies must focus on those resources and capabilities that are truly valuable: difficult to get by other companies, with a positive effect on the business, being irreplaceable for the company, highly complementary to other resources/capabilities, low accessible to competitors. At the same time, it is crucial to promote and strengthen the development of the own knowledge, leading to a distinctive identity and personality in the processes and activities the companies carry out. KM studies these changes, and the forecasting of them, trying to respond to the above mentioned challenges by designing and developing concepts, tools and management models.

Present study aims at analyzing the effect (at company and industrial sector levels) of some KM practices on competitiveness, success and survival. The study at the industrial sector level is important because both opportunities and menaces are the same for the companies in the sector. Among the analyzed practices, it is worth mentioning the competitive advantages, critical capabilities and pieces of knowledge, used resources, formalize and document the available knowledge, needed ways of learning, which one of those are more profitable, how do they have to design efficient strategies for knowledge transfer, share, and capitalization. To sum up, the study intends to generate interesting guidelines about some questions that have not been addressed up to now in the KM field and lead company activity to take advantage of KM. 
To do that, present research proposes the application of neural projection models $[1,4,5]$ (described in Sect. 2) to generate intuitive visualizations of KM data by reducing their dimensionality. Thanks to such a visualization, the inner structure of the dataset is revealed. A comprehensive analysis of such structure by a KM expert, leads to interesting conclusions about the KM status of both companies and industrial sectors. To validate the proposed tool, as a technological support for KM, it has been validated against two real-life datasets, that are related with two different industrial sectors; on the one hand the Electrical and Telecommunications industries and, on the other hand, Timber industries.

The proposed neural approach is presented in Sect. 2, while details about the data and the experiments are provided in Sect. 3. Section 4 presents the derived conclusions and proposed future work.

\section{Neural Visualization}

Projection models [6] operate on the spatial coordinates of high-dimensional data, in order to project them onto lower dimensional spaces. The main goal is to identify the patterns that exist across dimensional boundaries by identifying "interesting" directions, in terms of any specific index or projection. Such indexes or projections are, for example, based on the identification of directions that account for the largest variance of a data set -i.e. Principal Component Analysis (PCA) [7, 8] - or the identification of higher-order statistics such as the skew or kurtosis index -i.e. Exploratory Projection Pursuit (EPP) [6]. Having identified the most interesting projections, the data is then projected onto a lower dimensional subspace, plotted onto two or three dimensions, which makes it possible to examine its structure with the naked eye.

The combination of projection techniques together with the use of scatter plot matrices is a very useful visualization tool to investigate the intrinsic structure of multidimensional data sets, allowing experts to study the relations between different components, factors or projections, depending on the technique that is applied.

The solution proposed in this research applies an unsupervised neural model called Cooperative Maximum Likelihood Hebbian Learning (CMLHL) [4]. It is based on Maximum Likelihood Hebbian Learning (MLHL) [4], and introduces the application of lateral connections [4] derived from the Rectified Gaussian Distribution [9]. This connectionist model has been chosen because it reduces the data dimensionality while preserving the topology in the original data set. Considering an $N$-dimensional input vector $(x)$, and an $M$-dimensional output vector $(y)$, with $W_{i j}$ being the weight (linking input $j$ to output $i$ ), then CMLHL can be expressed as:

1. Feed-forward step:

$$
y_{i}=\sum_{j=1}^{\mathbf{N}} W_{i j} x_{j}, \forall i
$$


2. Lateral activation passing:

3. Feedback step:

$$
y_{i}(t+1)=\left[y_{i}(t)+\tau(b-A y)\right]^{+} .
$$

$$
e_{j}=x_{j}-\sum_{i=1}^{M} W_{i j} y_{i}, \forall j .
$$

4. Weight change:

$$
\Delta W_{i j}=\eta \cdot y_{i} \cdot \operatorname{sign}\left(e_{j}\right)\left|e_{j}\right|^{p-1} .
$$

where: $\eta$ is the learning rate, $\tau$ is the "strength" of the lateral connections, $b$ the bias parameter, $p$ a parameter related to the energy function and $A$ a symmetric matrix used to modify the response to the data [4]. The effect of this matrix is based on the relation between the distances separating the output neurons. This neural projection model has been previously applied to the KM field [1].

\section{Experiments \& Results}

As previously mentioned, neural visualization models have been applied to analyze data coming from companies in different industrial sectors. The analyzed data, as well as the obtained results are described in this section.

\subsection{Dataset}

For the proposed analysis, data coming from 44 companies in two different sectors (Electrical and Telecommunications industries, and Timber industries) have been collected. The headquarters of all the studied companies are located in Burgos (Spain). Many different features have been collected for each company, related to four main areas:

- Learning and Knowledge.

- KM Practices

- Contribution and Competitiveness

- Sector and company characteristics.

Data from those hour areas amount to 146 features. The complete analysis is too large for present paper so, only features related to the first area (learning and knowledge) are addressed. The 33 analyzed features are organized in some different subareas: competitive advantages, capabilities for competitive advantages, KM elements, ways of learning, knowledge documentation and given situations. Information about the features and their values is shown in Table 1. 
Table 1 Data features for each one of the companies in the dataset

\begin{tabular}{|c|c|c|}
\hline$\#$ & Feature name & Value range \\
\hline \multicolumn{3}{|c|}{ Competitive advantages } \\
\hline 1 & Product/service & 0 (absent) and 1 (present) \\
\hline 2 & Customer service & 0 (absent) and 1 (present) \\
\hline 3 & Innovation & 0 (absent) and 1 (present) \\
\hline 4 & Company's image/brand & 0 (absent) and 1 (present) \\
\hline 5 & Employees & 0 (absent) and 1 (present) \\
\hline 6 & Agility/adaptation & 0 (absent) and 1 (present) \\
\hline 7 & Technology & 0 (absent) and 1 (present) \\
\hline 8 & Company's management/organization & 0 (absent) and 1 (present) \\
\hline \multicolumn{3}{|c|}{ Capabilities for competitive advantages } \\
\hline 9 & Human resources & 0 (absent) and 1 (present) \\
\hline 10 & Knowledge/experience/know-how & 0 (absent) and 1 (present) \\
\hline 11 & Innovation/Competitiveness/Design & 0 (absent) and 1 (present) \\
\hline 12 & Organization & 0 (absent) and 1 (present) \\
\hline 13 & Company's nature & 0 (absent) and 1 (present) \\
\hline \multicolumn{3}{|c|}{ KM elements } \\
\hline 14 & Patents and brands & 0 (absent) and 1 (present) \\
\hline 15 & Processes & 0 (absent) and 1 (present) \\
\hline 16 & Experience & 0 (absent) and 1 (present) \\
\hline 17 & Technology & 0 (absent) and 1 (present) \\
\hline 18 & Innovation & 0 (absent) and 1 (present) \\
\hline
\end{tabular}

Ways of learning

\begin{tabular}{l|l|l}
\hline 19 & Several employees working together & 0 (absent) and 1 (present) \\
\hline 20 & Experienced employees working with unexperienced ones & 0 (absent) and 1 (present) \\
\hline 21 & Some employees get deep knowledge in specialized areas & 0 (absent) and 1 (present) \\
\hline 22 & Internal and external training courses & 0 (absent) and 1 (present) \\
\hline 23 & New duties are assumed by employees on a regular basis & 0 (absent) and 1 (present) \\
\hline 24 & $\begin{array}{l}\text { New employees, specialist in a certain issue, join the } \\
\text { company }\end{array}$ & 0 (absent) and 1 (present) \\
\hline 25 & Collaboration with competitor companies & 0 (absent) and 1 (present) \\
\hline 26 & Opinion of clients and suppliers is taken into account & 0 (absent) and 1 (present) \\
\hline 27 & External staff is subcontracted & 0 (absent) and 1 (present) \\
\hline \multicolumn{2}{l}{ Knowledge documentation } & 1 (low) to 4 (high) \\
\hline 28 & Level of knowledge documentation
\end{tabular}

\section{Given situations}

\begin{tabular}{l|l|l}
\hline 30 & $\begin{array}{l}\text { When a problem arises, the company knows who should } \\
\text { be contacted }\end{array}$ & 1 (no) to 4 (totally) \\
\hline 31 & New ideas and proposals are welcomed & 1 (no) to 4 (totally) \\
\hline 32 & $\begin{array}{l}\text { There are collaboration and knowledge exchange among } \\
\text { employees, clients and suppliers }\end{array}$ & 1 (no) to 4 (totally) \\
\hline 33 & Experienced employees teach the other ones & 1 (no) to 4 (totally) \\
\hline
\end{tabular}




\subsection{Results}

The obtained projections, together with the conclusions derived from them are described in this section. Each company in the dataset is depicted as a single point (clack square) in the figures below.

The PCA projection of the dataset is shown in Fig. 1. A clear structure of the dataset can not be identified from this projection as groups of data are not easily identified. However, the ordering of data in such a visualization has been analyzed by studying every single data. As a result, it can be said that companies in the right side of the projection are those with best positions regarding competitive advantages, capabilities, ways of learning and given situations. Complementarily, companies in the left side of the projection are those in the worst situations, getting worse and worse while moving down, to the bottom of the projection. These are companies with only one competitive advantage, only one capability, and the ways of learning and knowledge documentation are poor.

Fig. 1 PCA projection of the dataset

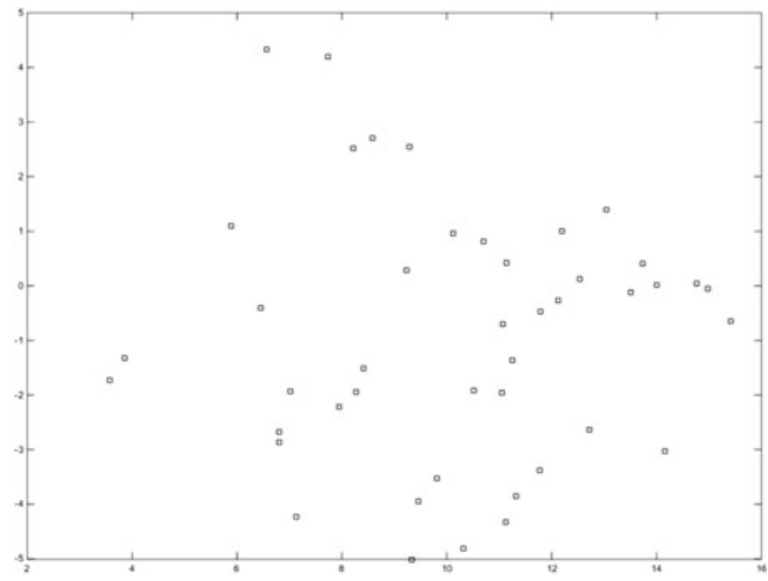

Figure 2 shows the projection of the dataset by CMLHL. According to the location of each data (company) in the projection, some conclusions arise, as described below.

From the perspective of the "competitive advantages" group of features, it has been checked that those data located in the first upper third of Fig. 2 represent companies with all the competitive advantages proposed in present study. In the middle of the projection, CMLHL places those companies with about 3 or 4 of the competitive advantages (product/service, customer service, employees, and company's management/organization). Those companies at the bottom only attain one competitive advantage: agility/adaptation (left side) or Innovation (right side). The meaning of this ordering is that the companies in the upper right third of Fig. 2 (groups 2.1 and 2.2) are those that have been able to develop and apply knowledge 
for the creation of an important amount of competitive advantages. From top to bottom, the companies are ordered according to this amount of competitive advantages. By applying CMLHL, it can be easily identified those companies that excel in applying knowledge to get competitive advantages. Additionally, there are some other companies in the way to get all the advantages or some other elements to improve their competitiveness.

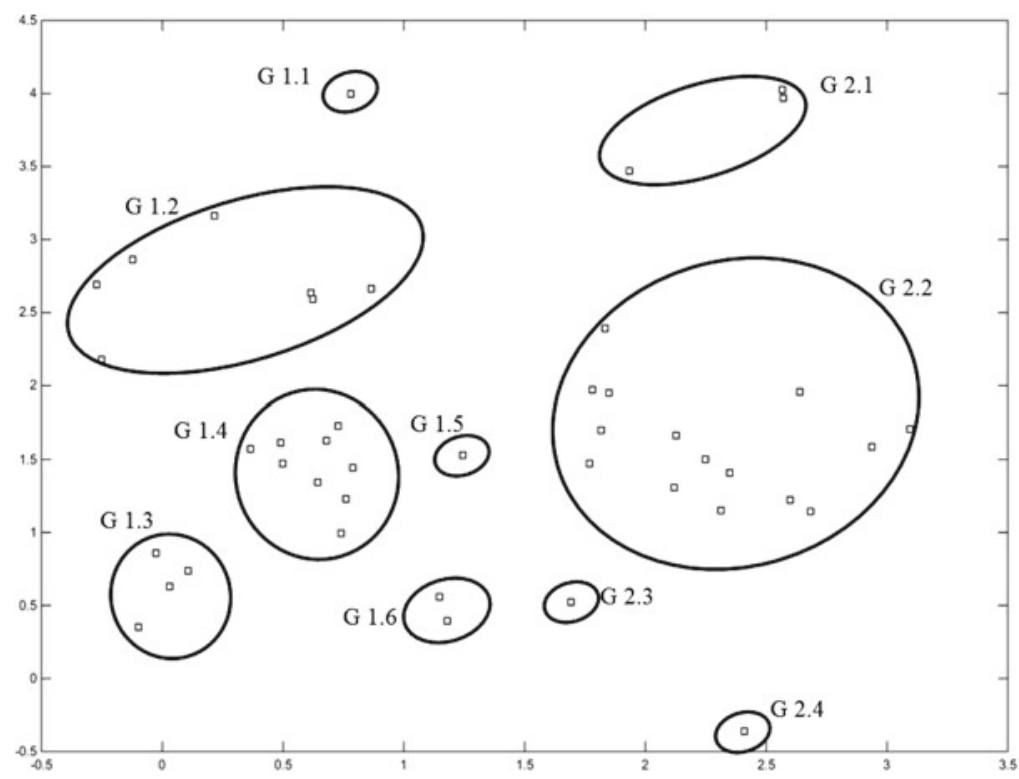

Fig. 2 CMLHL projection of the dataset

With regard to the "capabilities for competitive advantages" group of features, the ordering is similar to the one for "competitive advantages" but, in this case, it is more clearly defined. All the companies in the upper right third of Fig. 2 are those with the following capabilities: "Human resources", "Knowledge/experience/know-how" and "Organization". From the top to the bottom of the figure, some of these capabilities are not present ("Knowledge/experience/know-how"). At the very bottom, only the "Organization" capability is present. There is a similar situation in the left side of the figure; companies in the top left side are those with the "Knowledge/experience/ know-how" and "Innovation/Competitiveness/Design" features. From the top to the bottom of the figure, some of these capabilities are not present. Most companies are in intermediate positions. However, 16 of them are different from the other ones because at least three capabilities are present. From a combined analysis of the "competitive advantages" and "capabilities for competitive advantages" features, it can be said that the more competitive advantages, the greater capabilities for the company. The preferably capabilities are "Human resources", "Knowledge/experience/know-how", and "Organization". 
The third analyzed group of features is "KM elements", that is related to the meaning of KM for the company. The ordering is similar to that of the two previous groups of features (top to bottom and right to left). Companies in the upper right third of Fig. 2 consider that KM consists of at least four elements ("Processes", "Experience", "Technology", and "Innovation"). It is then easy to identify that companies in the right side of Fig. 2 relate the KM to more elements than those in the left side.

After analyzing the "Ways of learning" group of features, it can be said that once again, there is a clear ordering of data. It is similar to that for the three previous groups of features; all the different ways of learning, except "Collaboration with competitor companies" and "External staff is subcontracted", are present for companies in the upper right third of Fig. 2. In the intermediate region of the figure, the feature "New employees, specialist in a certain issue, join the company" is not present. At the bottom, only the "Opinion of clients and suppliers is taken into account" is present.

As a conclusion for this group of features, it can be said that those companies applying more and new ways of learning are located in better positions (upper right third of the figure). Those companies are the best ones for the analyzed groups of features: "Competitive advantages", "Capabilities for competitive advantages", and "KM elements".

For the "Given situations" group of features, the ordering is similar to the previous ones; companies in the upper right third of Fig. 2 get the highest scores for these features. On the contrary, CMLHL does not provide a clear ordering for the feature "Knowledge documentation" as companies in the best situations for such feature are located in opposite groups (1.1 and 2.4). Group 2.4 (with only one company) can be considered as a special case because its situation for the competitive advantages and capabilities is good but there are some weaknesses regarding the ways of learning and the given situations.

To sum up the conclusions related to Fig. 2, it can be said that the projection is coherent with regard to most of the analyzed groups of features. The companies with many competitive advantages, do have capabilities that support the advantages, what leads to innovative ways of learning to generate new ideas and apply new knowledge. The main goal is to improve/development of products, services, functions, processes, etc. which state the competitiveness and the survival of the company. In a more precise way, it can be said that the companies in the best situation from the KM perspective are gathered in groups 2.1 and 2.2, while those companies in the worst position are placed in group 1.3. All the other ones are in intermediate positions.

After the previous analysis (at a company level), a more general analysis (at an industrial sector level) has been carried out. To the best of the authors knowledge, this is the first time that neural models are applied to the analysis of KM along different industrial sectors. The CMLHL projection of the data has been modified to distinguish between companies belonging to one sector or the other one. Thus, in Fig. 3, Electrical and Telecommunications industries are depicted as black stars, while Timber industries are depicted as red circles.

From Fig. 3, it can be said that those companies belonging to the Electrical and Telecommunications sector are mainly located in the right side of the projection. 


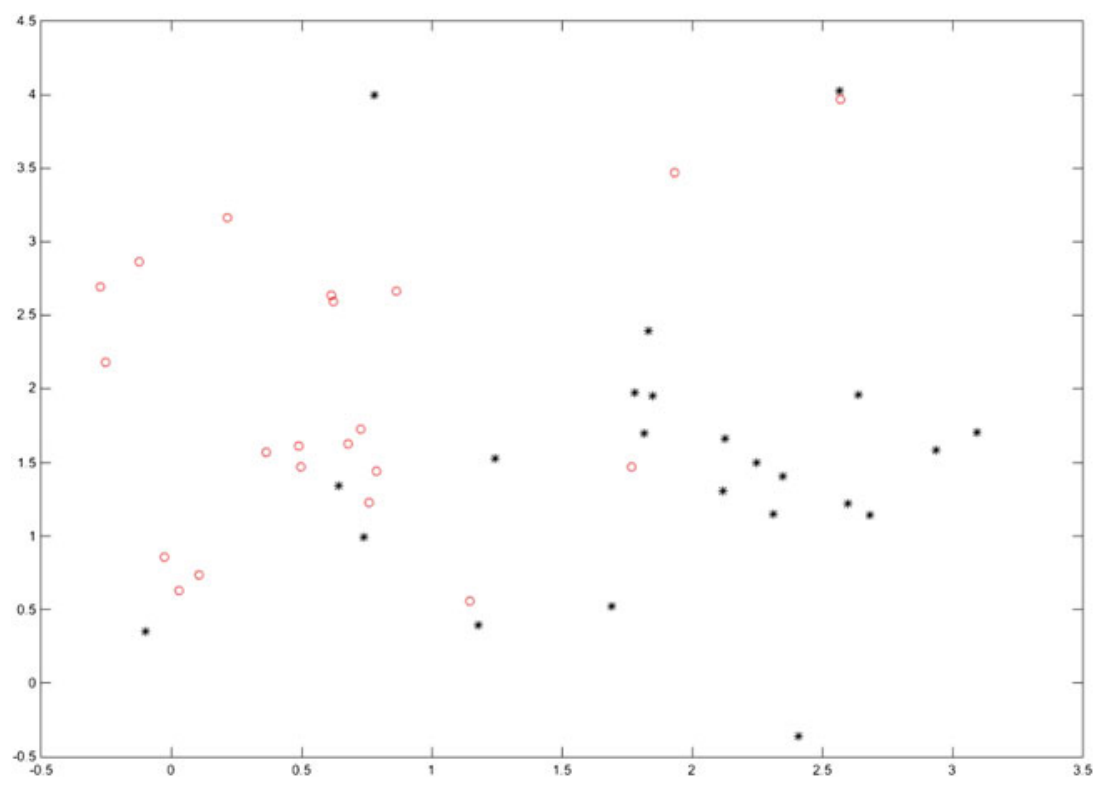

Fig. 3 CMLHL projection of the retention factor dataset

Those in best positions are located in group 2.2, standing out because of their competitive advantages, ways of learning and given situations. On the other hand, companies belonging to the Timber sector are mainly located in the left side of the projection. Group 1.2 contains companies that stand out because of the ways of learning and the behavior to employ and increase the knowledge. Similarly, group 1.4 contains those companies from the timber sector that excel in distinctive capabilities, KM elements, and the given situations.

Generally, it can be said that there are not strong differences between the two analysed industrial sectors. Taking into account all the companies belonging to each one of them, it can be said that their status is almost similar, although it could be defined as slightly better for the Electrical and Telecommunications sector. This can be easily seen in Group 2.2 that is one of those gathering companies in the best situations. All the companies in such groups, expect one, belong to the Electrical and Telecommunications sector.

\section{Conclusions and Future Work}

The main objective for present research was two-fold: on the one hand, to check neural projection models for the intuitive visualization of qualitative, abstract and disordered data associated to KM in different industrial sectors. More precisely, the analysis is focused on data related to Learning and Knowledge, for a significant 
real-life dataset in two industrial sectors: Electrical and Telecommunications industries and Timber industries. On the other hand, present research proposes technological support to bridge the gap between theoretical KM formulations to practical tools that support KM experts in diagnosis and decision taking.

This objective has been met as CMLHL has ordered and grouped the unknown dataset under analysis. After a thorough subsequent study, it has been proved that the obtained projection is coherent with the data and meaningful. Hence, it can support decision taking aimed at improving effectiveness of KM practices, ways of learning and behaviors to assure a proper KM in companies. Among the obtained results, the following ones are worth mentioning:

- The precise location of companies in CMLHL projection according to certain features determines the level of the companies according to their configuration of Learning and Knowledge, included in the KM. This is interesting to know the situation related to a certain feature for a company and take corresponding decisions. More precisely, obtained results let us know whether a certain company has competitive advantages, and which capabilities are supporting them. Similarly, the ways of learning that the company applies to guarantee and make profitable the knowledge it has or need to acquire, the behavior or attitude of employees in usual situations related to KM, such as problem solving, expert querying, encouragement of new ideas, or sharing, teaching and exchanging the existent knowledge.

- The convergence of some features, with certain values, in areas clearly identified, that represents industrial situations of a diverse nature, ranging from very good to extremely bad. These groups of data, located in a certain area of the projection, allow for not only informing about the situation of the company regarding Learning and Knowledge, but also, deploying a wide variety of actions for moving to the best locations and leaving those that are unrecommendable or inefficient.

- The support for cross-sector analysis of industrial companies in a way that general conclusions about a certain sector can be drawn from the projection of the companies belonging to it.

Consequently, the applied neural model supports the easy and meaningful analysis of the features associated to Learning and Knowledge. Furthermore, interesting conclusions can be obtained about how to evolve and progress towards the deployment and execution of efficient $\mathrm{KM}$ in industrial companies from the Electrical and Telecommunications and Timber sectors.

For future work, it is proposed to extend present study to cover some other features associated to KM, and some more sectors and companies. Additionally, some other neural projection models could be applied to obtain more interesting visualizations of data. 


\section{References}

1. Herrero Á, Corchado E, Sáiz L, Abraham A (2010) DIPKIP: A connectionist knowledge management system to identify knowledge deficits in practical cases. Comput Intell 26:26-56

2. Durst S, Edvardsson IR (2012) Knowledge management in SMEs: a literature review. J Knowl Manag 16:879-903

3. Levy M (2011) Knowledge retention: minimizing organizational business loss. J Knowl Manag 15:582-600

4. Herrero Á, Corchado E, Jiménez A (2011) Unsupervised neural models for country and political risk analysis. Expert Syst Appl 38:13641-13661

5. Herrero Á, Corchado E, Gastaldo P, Zunino R (2009) Neural projection techniques for the visual inspection of network traffic. Neurocomputing 72:3649-3658

6. Friedman JH, Tukey JW (1974) A projection pursuit algorithm for exploratory data-analysis. IEEE Trans Comput 23:881-890

7. Hotelling H (1933) Analysis of a complex of statistical variables into principal components. J Educ Psychol 24:417-444

8. Pearson K (1901) On lines and planes of closest fit to systems of points in space. Phil Mag 2:559-572

9. Seung HS, Socci ND, Lee D (1998) The Rectified Gaussian Distribution. Adv Neural Inf Process Syst 10:350-356 